\title{
SECOND-ORDER SYSTEMS WITH SINGULAR MASS MATRIX AND AN EXTENSION OF GUYAN REDUCTION*
}

\author{
SANJAY P. BHAT ${ }^{\dagger}$ AND DENNIS S. BERNSTEIN ${ }^{\dagger}$
}

\begin{abstract}
The set of consistent initial conditions for a second-order system with singular mass matrix is obtained. In general, such a system can be decomposed (i.e., partitioned) into three coupled subsystems of which the first is algebraic, the second is a regular system of first-order differential equations, and the third is a regular system of second-order differential equations. Under specialized conditions, these subsystems are decoupled. This result provides an extension of Guyan reduction to include viscous damping.
\end{abstract}

Key words. second-order differential equation, singular mass matrix

AMS subject classifications. 34A30, 70J05, 93A99

$\quad$ Notation.
$\mathcal{R}(\mathcal{C})$
$\mathcal{R}^{n}\left(\mathcal{R}^{n \times n}\right)$
$(A)_{i j}$
$\operatorname{rank} A(\operatorname{def} A$, ind $A)$
$\mathcal{N}(A)(\mathcal{R}(A))$
$A^{\mathrm{T}}$
$A>(\geq) 0$
$\mathcal{S}_{1} \perp \mathcal{S}_{2}$
$\mathcal{S}_{1} \oplus \mathcal{S}_{2}$
$\mathcal{S}_{1} \cap \mathcal{S}_{2}$
$\triangleq$

real (complex) numbers, real vectors (matrices) of dimension $n(n \times n)$, $i j$ th element of the matrix $A$, rank (defect, index) of the matrix $A$, nullspace (range) of the matrix $A$, transpose of the matrix $A$, symmetric positive- (nonnegative-) definite matrix, subspace $\mathcal{S}_{1}$ orthogonal to the subspace $\mathcal{S}_{2}$, direct sum of the subspaces $\mathcal{S}_{1}$ and $\mathcal{S}_{2}$, intersection of the subspaces $\mathcal{S}_{1}$ and $\mathcal{S}_{2}$, equal by definition.

1. Introduction. Singular linear systems, that is, linear systems of the form $E \dot{x}=A x$, where the matrix $E$ is singular, have been studied extensively. Such systems arise in singular perturbation problems [1], optimal control [2], and large scale interconnected systems and economics [3].

An interesting property of singular systems is the existence of impulsive behavior for certain initial conditions. Although for consistent initial conditions the system behaves like a regular linear system, initial conditions that are not consistent lead to impulsive behavior by which the state is instantaneously transferred to the set of consistent initial conditions. A familiar example is the sparking that often occurs when two electrical subsystems are suddenly connected together.

In the present paper we study the matrix second-order equation $M \ddot{q}+C \dot{q}+K q=$ 0 , where $M, C$, and $K$ denote nonnegative-definite mass, damping, and stiffness matrices, respectively. This equation represents a special case of a singular system when the mass matrix $M$ is singular. A second-order system with singular mass matrix may arise from a singular perturbation problem [4] or may represent a large scale system with algebraic constraints placed on the state variables of the component subsystems. Our goal is to investigate the properties of this second-order equation in the case in which $M$ is singular.

* Received by the editors May 20, 1994; accepted for publication (in revised form) by C. Meyer October 3, 1995. This research was supported in part by Air Force Office of Scientific Research grant F49620-95-1-0019.

† Department of Aerospace Engineering, University of Michigan, Ann Arbor, MI 48109-2118 (dsbaero@engin.umich.edu). 
In applications it is often the case that $M$ is not singular but rather contains terms that are numerically small. It is then standard engineering practice to assume that these terms are zero, in which case $M$ is singular. However, if the initial conditions are restricted to lie in the set of consistent initial conditions, then the behavior of the system is governed by a regular system of linear differential equations of reduced dimension. This is the idea behind Guyan reduction [5], which is a modelreduction technique widely used for analyzing structural vibrations of large undamped mechanical systems. Certain finite element modeling techniques involving massless coordinates may also give rise to second-order models with singular mass matrices [6, pp. 107-109]. Although numerical simulations of such systems can be problematic because of the impulsive behavior of the model, such problems can be avoided by restricting the initial conditions appropriately. Singular mass matrices also arise in nonlinear multi-degree-of-freedom mechanical systems [7].

The purpose of this paper is to determine the set of consistent initial conditions for matrix second-order systems with nonnegative-definite mass, damping, and stiffness matrices and to construct a reduced model for such systems when the states are restricted to lie in this set. These results are obtained by specializing known results relating to singular systems to the second-order case. It is shown that a secondorder system can be decomposed (i.e., partitioned) into three coupled subsystems of equations - the first is algebraic, the second is a regular system of first-order differential equations, and the third is a regular system of second-order differential equations. This result is used to obtain an extension of Guyan reduction to include viscous damping.

2. Preliminaries. We begin by introducing some definitions concerning the linear system

$$
E \dot{x}(t)=A x(t), x(0)=c,
$$

where $t \geq 0, x(t) \in \mathcal{R}^{n}, E, A \in \mathcal{R}^{n \times n}$, and where $E$ may be singular. In the definitions to follow, a solution is assumed to be analytic. In general, the singular system (1) admits nonanalytic solutions in the form of distributions [8].

A vector $c \in \mathcal{R}^{n}$ is a consistent initial condition if the initial value problem (1) possesses at least one solution. It is easy to see that the set of consistent initial conditions of (1) is a linear subspace. The system (1) is tractable if the initial value problem (1) possesses exactly one solution for every consistent initial condition $c$. The following proposition, which is stated and proved as Theorem 9.2.1 in [9], gives a necessary and sufficient condition for (1) to be tractable.

Proposition 1. The system (1) is tractable if and only if there exists $\lambda \in \mathcal{C}$ such that $\operatorname{rank}(\lambda E-A)=n$.

If $\lambda \in \mathcal{C}$ and $\operatorname{rank}(\lambda E-A)=n$, then we define $\hat{E}_{\lambda} \triangleq(\lambda E-A)^{-1} E$. Recall that the index of a matrix $A$, denoted by ind $A$, is the smallest nonnegative integer $k$ such that rank $A^{k}=\operatorname{rank} A^{k+1}$. The following lemma gives some properties of $\hat{E}_{\lambda}$ that are independent of $\lambda$ whenever $\hat{E}_{\lambda}$ is defined. This lemma is stated and proved as Theorem 9.2.2 in [9].

LEMma 1. Suppose $\lambda_{1}, \lambda_{2} \in \mathcal{C}$ satisfy $\operatorname{rank}\left(\lambda_{1} E-A\right)=\operatorname{rank}\left(\lambda_{2} E-A\right)=n$. Then ind $\hat{E}_{\lambda_{1}}=$ ind $\hat{E}_{\lambda_{2}}$ and $\mathcal{R}\left(\hat{E}_{\lambda_{1}}^{k}\right)=\mathcal{R}\left(\hat{E}_{\lambda_{2}}^{k}\right)$, where $k=$ ind $\hat{E}_{\lambda_{1}}$.

The following proposition, which follows from Theorem 9.2.3 of [9], characterizes the set of consistent initial conditions of (1).

Proposition 2. Suppose that (1) is tractable, let $\lambda \in \mathcal{C}$ be such that $\operatorname{rank}(\lambda E-$ 
$A)=n$, and let $k=$ ind $\hat{E}_{\lambda}$. Then the set of consistent initial conditions of (1) is given by $\mathcal{R}\left(\hat{E}_{\lambda}^{k}\right)$.

3. Second-order systems with singular mass matrix. In this section, the results stated in the previous section are specialized to the matrix second-order system

$$
M \ddot{q}+C \dot{q}+K q=0,
$$

where $q \in \mathcal{R}^{r}$ and $M, C, K \in \mathcal{R}^{r \times r}$ denote symmetric nonnegative-definite mass, damping, and stiffness matrices, respectively. This system can be rewritten in the first-order form (1) by defining

$$
x \triangleq\left[\begin{array}{c}
q \\
\dot{q}
\end{array}\right], E \triangleq\left[\begin{array}{cc}
I & 0 \\
0 & M
\end{array}\right], A \triangleq\left[\begin{array}{cc}
0 & I \\
-K & -C
\end{array}\right] .
$$

Note that if the mass matrix $M$ is singular then $E$ is also singular.

Before proceeding further, we state the following useful lemma.

Lemma 2. Suppose that $P, Q \in \mathcal{R}^{r \times r}$ and $P \geq 0$ and $Q \geq 0$. Then $\mathcal{N}(P+Q)=$ $\mathcal{N}(P) \cap \mathcal{N}(Q)$ and

$$
\operatorname{rank}(P+Q)=\operatorname{rank}\left[\begin{array}{c}
P \\
Q
\end{array}\right]
$$

The following theorem is an application of Proposition 1 to (2).

THEOREM 1. The system (2) is tractable if and only if $M+C+K>0$. In this case, $E-A$ is invertible.

Proof. Since

$$
\lambda E-A=\left[\begin{array}{cc}
I & 0 \\
-(\lambda M+C) & I
\end{array}\right]\left[\begin{array}{cc}
0 & -I \\
\lambda^{2} M+\lambda C+K & 0
\end{array}\right]\left[\begin{array}{cc}
I & 0 \\
-\lambda I & I
\end{array}\right],
$$

it follows that

$$
\operatorname{rank}(\lambda E-A)=r+\operatorname{rank}\left(\lambda^{2} M+\lambda C+K\right) .
$$

If $M+C+K>0$, then $\operatorname{rank}\left(\lambda^{2} M+\lambda C+K\right)=r$ for $\lambda=1$. The result now follows from (3) and Proposition 1.

Conversely, suppose there exists nonzero $x \in \mathcal{R}^{r}$ such that $(M+C+K) x=0$. Then, since $M, C$, and $K$ are nonnegative definite, it follows from Lemma 1 that $M x=C x=K x=0$. Thus $\left(\lambda^{2} M+\lambda C+K\right) x=0$ for every $\lambda \in \mathcal{C}$. Consequently, (3) implies that $\operatorname{rank}(\lambda E-A)<2 r$ for every $\lambda \in \mathcal{C}$. It now follows from Proposition 1 that (2) is not tractable, as required.

If $M+C+K>0$, then it follows from (3) that $\operatorname{rank}(E-A)=r+\operatorname{rank}(M+$ $C+K)=2 r$ so that $E-A$ is invertible.

Since we are interested only in systems possessing unique solutions, we shall assume that $M+C+K>0$ throughout the rest of this paper. In this case, it follows from Theorem 1 that the matrix $E-A$ is invertible. We define $\hat{M} \triangleq M+C+K$ and $\hat{E} \triangleq(E-A)^{-1} E$. Note that

$$
\hat{E}=\left[\begin{array}{cc}
\hat{M}^{-1}(M+C) & \hat{M}^{-1} M \\
-\hat{M}^{-1} K & \hat{M}^{-1} M
\end{array}\right]
$$

The following lemma gives a few properties of $\hat{E}$. 
LEMMA 3. The matrix $\hat{E}$ satisfies $\operatorname{rank} \hat{E}^{2}=\operatorname{rank} M+\operatorname{rank}(M+C)$. Furthermore, the following statements are valid.

i) ind $\hat{E} \leq 2$.

ii) ind $\hat{E} \leq 1$ if and only if $M+C>0$.

iii) ind $\hat{E}=0$ if and only if $M>0$.

Proof. Let

$$
y_{k}=\left[\begin{array}{l}
y_{k 1} \\
y_{k 2}
\end{array}\right]
$$

and $y_{k+1} \triangleq \hat{E}^{k+1} y_{k}$ for $k=0,1,2$, where $y_{k 1}, y_{k 2} \in \mathcal{R}^{r}$ for $k=0,1,2,3$. Now, suppose $\hat{E}^{3} y_{0}=\hat{E}^{2} y_{1}=\hat{E} y_{2}=y_{3}=0$. Then $E y_{2}=(E-A) y_{3}=0$, that is,

$$
y_{21}=\hat{M}^{-1}\left[(M+C) y_{11}+M y_{12}\right]=0
$$

and

$$
M y_{22}=M \hat{M}^{-1}\left(-K y_{11}+M y_{12}\right)=0 .
$$

Therefore $0=M y_{21}-M y_{22}=M y_{11}$. Premultiplying (4) by $y_{11}^{\mathrm{T}} \hat{M}$ yields $y_{11}^{\mathrm{T}} C y_{11}=0$. Since $C$ is nonnegative definite, it follows that $C y_{11}=0$. Using $(M+C) y_{11}=0$ in (4) gives $M y_{12}=0$. Thus

$$
M y_{11}=C y_{11}=M y_{12}=0
$$

Note that in deriving (6), no use was made of the fact that $y_{1}=\hat{E} y_{0}$. Thus it is true in general that $\hat{E}^{2} y_{1}=0$ implies

$$
\left[\begin{array}{cc}
C & M \\
M & 0
\end{array}\right] y_{1}=0 .
$$

The converse can be easily verified. Thus

$$
\mathcal{N}\left(\hat{E}^{2}\right)=\mathcal{N}\left[\begin{array}{cc}
C & M \\
M & 0
\end{array}\right]
$$

Hence

$$
\operatorname{rank} \hat{E}^{2}=\operatorname{rank} M+\operatorname{rank}\left[\begin{array}{c}
C \\
M
\end{array}\right] \text {. }
$$

Since $M$ and $C$ are nonnegative definite, it follows from Lemma 1 that rank $\hat{E}^{2}=$ rank $M+\operatorname{rank}(M+C)$.

To prove i) it suffices to show that $\mathcal{N}\left(\hat{E}^{3}\right) \subseteq \mathcal{N}\left(\hat{E}^{2}\right)$. Using (6) we compute $y_{11}^{\mathrm{T}} \hat{M} y_{11}=y_{01}^{\mathrm{T}}(M+C) y_{11}+y_{02}^{\mathrm{T}} M y_{11}=0$. Since $\hat{M}$ is positive definite, it follows that $y_{11}=0$. This together with (6) implies that $y_{22}=\hat{M}^{-1}\left(-K y_{11}+M y_{12}\right)=0$. It now follows from (4) that $y_{2}=0$. Thus $\hat{E}^{3} y=0$ implies that $\hat{E}^{2} y=0$. This proves i).

To prove ii) note that the index of $\hat{E}$ is less than 2 if and only if $\operatorname{rank} \hat{E}^{2}=\operatorname{rank} \hat{E}$. Since $\operatorname{rank} \hat{E}^{2}=\operatorname{rank} M+\operatorname{rank}(M+C)$ and $\operatorname{rank} \hat{E}=\operatorname{rank} E=\operatorname{rank} M+r$, it follows that ind $\hat{E}<2$ if and only if $M+C$ is positive definite.

Finally, ind $\hat{E}=0$ if and only if rank $\hat{E}=2 r$. Since rank $\hat{E}=\operatorname{rank} E=$ $r+\operatorname{rank} M$, it follows that rank $\hat{E}=2 r$ if and only if $M$ is positive definite. 
The following theorem uses Lemma 3 to determine the set of consistent initial conditions of (2).

THEOREM 2. The set of consistent initial conditions of (2) is given by $\mathcal{R}\left(\hat{E}^{2}\right)$. Furthermore, if $M+C>0$, then the set of consistent initial conditions of (2) is given by $\mathcal{R}(\hat{E})$.

Proof. The results follow from Proposition 2 and Lemma 3.

The second part of Theorem 2 is a special case of Proposition 8.2.1 in [10].

COROLlaRY 1. The dimension of the subspace of consistent initial conditions of (2) is $\operatorname{rank} M+\operatorname{rank}(M+C)$.

Proof. The result follows from Theorem 2 and Lemma 3.

4. Model reduction. In this section it is shown that the second-order system (2) can be decomposed into a system of algebraic equations, a regular first-order system of differential equations, and a regular second-order system of differential equations. It is also shown that under special assumptions the algebraic subsystem can be eliminated to obtain a regular second-order system having fewer degrees of freedom.

For convenience, define $r_{1} \triangleq \operatorname{def}(M+C), r_{2} \triangleq \operatorname{def} M-r_{1}$, and $r_{3} \triangleq \operatorname{rank} M$. Note that $r_{1}+r_{2}+r_{3}=r$. It can be seen from Corollary 1 that the subspace of consistent initial conditions has dimension $2 r_{3}+r_{2}$. In this section we assume that $M$ is singular but nonzero, in which case $r_{1}+r_{2}>0$ and $r_{3}>0$.

Theorem 3. i) Suppose $\operatorname{def} M>\operatorname{def}(M+C)>0$. Then there exists an orthogonal matrix $U \in \mathcal{R}^{r \times r}$ such that

$$
\begin{gathered}
U^{\mathrm{T}} M U=\left[\begin{array}{ccc}
0 & 0 & 0 \\
0 & 0 & 0 \\
0 & 0 & M_{3}
\end{array}\right], U^{\mathrm{T}} C U=\left[\begin{array}{ccc}
0 & 0 & 0 \\
0 & C_{2} & C_{23} \\
0 & C_{23}^{\mathrm{T}} & C_{3}
\end{array}\right], \\
U^{\mathrm{T}} K U=\left[\begin{array}{ccc}
K_{1} & K_{12} & K_{13} \\
K_{12}^{\mathrm{T}} & K_{2} & K_{23} \\
K_{13}^{\mathrm{T}} & K_{23}^{\mathrm{T}} & K_{3}
\end{array}\right],
\end{gathered}
$$

where $M_{3} \in \mathcal{R}^{r_{3} \times r_{3}}, C_{2} \in \mathcal{R}^{r_{2} \times r_{2}}$, and $K_{1} \in \mathcal{R}^{r_{1} \times r_{1}}$ are positive definite. Furthermore, $K_{12}=0, K_{2}=0$, and $K_{23}=0$ if and only if $\mathcal{N}(M+C) \perp \mathcal{N}(M+K)$ and $\mathcal{N}(M)=\mathcal{N}(M+C) \oplus \mathcal{N}(M+K)$.

ii) Suppose $\operatorname{def} M=\operatorname{def}(M+C)>0$. Then there exists an orthogonal matrix $U \in \mathcal{R}^{r \times r}$ such that

$$
U^{\mathrm{T}} M U=\left[\begin{array}{cc}
0 & 0 \\
0 & M_{2}
\end{array}\right], U^{\mathrm{T}} C U=\left[\begin{array}{cc}
0 & 0 \\
0 & C_{2}
\end{array}\right], U^{\mathrm{T}} K U=\left[\begin{array}{cc}
K_{1} & K_{12} \\
K_{12}^{\mathrm{T}} & K_{2}
\end{array}\right],
$$

where $M_{2} \in \mathcal{R}^{r_{3} \times r_{3}}$ and $K_{1} \in \mathcal{R}^{r_{1} \times r_{1}}$ are positive definite.

iii) Suppose $\operatorname{def} M>\operatorname{def}(M+C)=0$. Then there exists an orthogonal matrix $U \in \mathcal{R}^{r \times r}$ such that

$$
U^{\mathrm{T}} M U=\left[\begin{array}{cc}
0 & 0 \\
0 & M_{2}
\end{array}\right], U^{\mathrm{T}} C U=\left[\begin{array}{cc}
C_{1} & C_{12} \\
C_{12}^{\mathrm{T}} & C_{2}
\end{array}\right],
$$

where $M_{2} \in \mathcal{R}^{r_{3} \times r_{3}}$ and $C_{1} \in \mathcal{R}^{r_{2} \times r_{2}}$ are positive definite.

Proof. i) In this case, $r_{1}>0$ and $r_{2}>0$. Let $x_{1}, x_{2}, \ldots, x_{r}$ be an orthonormal basis for $\mathcal{R}^{r}$ such that $x_{1}, x_{2}, \ldots, x_{r_{1}+r_{2}}$ is an orthonormal basis for $\mathcal{N}(M)$ and $x_{1}, x_{2}, \ldots, x_{r_{1}}$ is an orthonormal basis for $\mathcal{N}(M+C)$. Let $U=$ 
$\left[\begin{array}{llll}x_{1} & x_{2} & \ldots & x_{r}\end{array}\right]$. Then it can easily be verified that $U^{\mathrm{T}} U=I$. Note that for every matrix $P,\left(U^{\mathrm{T}} P U\right)_{i j}=x_{i}^{\mathrm{T}} P x_{j}$. The sizes and placement of the zero subblocks in $U^{\mathrm{T}} M U$ and $U^{\mathrm{T}} C U$ now follow from the choice of the vectors $x_{1}, x_{2}, \ldots, x_{r}$. Since $M_{3}, C_{2}$, and $K_{1}$ are principal submatrices of the nonnegative-definite matrices $U^{\mathrm{T}} M U, U^{\mathrm{T}} C U$, and $U^{\mathrm{T}} K U$, respectively, it follows that $M_{3}, C_{2}$, and $K_{1}$ are nonnegative definite. Now rank $M_{3}=\operatorname{rank} M=r_{3}$, which is also the dimension of $M_{3}$. Hence $M_{3}>0$. To show that $C_{2}>0$, suppose that $C_{2} y_{2}=0$ for some $y_{2} \in \mathcal{R}^{r_{2}}$. Then $y_{2}^{\mathrm{T}} C_{2} y_{2}=z^{\mathrm{T}} C z=0$, where $z=U\left[\begin{array}{lll}0 & y_{2}^{\mathrm{T}} & 0\end{array}\right]^{\mathrm{T}}$. The nonnegative definiteness of $C$ leads to $C z=0$. Also $U^{\mathrm{T}} M z=0$. Thus $(M+C) z=0$. By construction, every vector in $\mathcal{N}(M+C)$ is of the form $U\left[\begin{array}{lll}y_{1}^{\mathrm{T}} & 0 & 0\end{array}\right]^{\mathrm{T}}$, where $y_{1} \in \mathcal{R}^{r_{1}}$. Therefore, $y_{2}=0$ and hence $C_{2}>0$. Finally, $K_{1}$ is a principal submatrix of the positive-definite matrix $U^{\mathrm{T}}(M+C+K) U$ and hence positive definite. This proves the first part of i).

If $\mathcal{N}(M+C) \perp \mathcal{N}(M+K)$ and $\mathcal{N}(M)=\mathcal{N}(M+C) \oplus \mathcal{N}(M+K)$, then the vectors $x_{r_{1}+1}, x_{r_{1}+2}, \ldots, x_{r_{1}+r_{2}}$ form a basis for $\mathcal{N}(M+K)$. By Lemma 1 , these vectors also lie in $\mathcal{N}(K)$. Since every element of $K_{12}, K_{2}$, and $K_{23}$ is of the form $x_{i}^{\mathrm{T}} K x_{j}$, where either $r_{1}+1 \leq i \leq r_{1}+r_{2}$ or $r_{1}+1 \leq j \leq r_{1}+r_{2}$, it follows that $K_{12}=0, K_{2}=0$, and $K_{23}=0$. If $K_{12}=0, K_{2}=0$, and $K_{23}=0$, then since $M_{3}$ and $K_{1}$ are positive definite, it follows that $\mathcal{N}(M+K)$ consists of vectors of the form $z=U\left[\begin{array}{lll}0 & y_{2}^{\mathrm{T}} & 0\end{array}\right]^{\mathrm{T}}$, where $y_{2} \in \mathcal{R}^{r_{2}}$. Thus the vectors $x_{r_{1}+1}, x_{r_{1}+2}, \ldots, x_{r_{1}+r_{2}}$ form a basis for $\mathcal{N}(M+K)$ and the result follows.

The proofs of ii) and iii) are similar.

Theorem 3 gives conditions under which $M, C$, and $K$ may be assumed without loss of generality to be of the form given by (7). Note that the first $r_{1}$ equations are algebraic while the remaining equations represent a regular first-order system of dimension $r_{2}$ coupled with a regular $r_{3}$-degree-of-freedom second-order system. The following corollary shows that under special assumptions the algebraic equations can be eliminated to obtain a regular second-order system with a reduced number of degrees of freedom.

Corollary 2. Suppose $\operatorname{def} M>\operatorname{def}(M+C)>0$ and assume that $\mathcal{N}(M+C) \perp$ $\mathcal{N}(M+K)$ and $\mathcal{N}(M)=\mathcal{N}(M+C) \oplus \mathcal{N}(M+K)$. Then there exists a matrix $S \in \mathcal{R}^{r \times r_{3}}$ such that $S^{\mathrm{T}} M S>0, S^{\mathrm{T}} C S \geq 0$, and $S^{\mathrm{T}} K S \geq 0$.

Proof. Under the stated assumptions, there exists a matrix $U \in \mathcal{R}^{r \times r}$ such that $U^{\mathrm{T}} M U, U^{\mathrm{T}} C U$, and $U^{\mathrm{T}} K U$ are given by (7) with $K_{12}=0, K_{2}=0$, and $K_{23}=0$. Define $S \in \mathcal{R}^{r \times r_{3}}$ by

$$
S=U\left[\begin{array}{c}
-K_{1}^{-1} K_{13} \\
-C_{2}^{-1} C_{23} \\
I
\end{array}\right]
$$

Then $S^{\mathrm{T}} M S=M_{3}$ is positive definite by Theorem 3 , and $S^{\mathrm{T}} C S$ and $S^{\mathrm{T}} K S$ are nonnegative definite since $C$ and $K$ are nonnegative definite.

The following corollary gives another case in which a reduction in the number of degrees of freedom can be achieved.

Corollary 3. Suppose $\operatorname{def} M=\operatorname{def}(M+C)>0$. Then there exists a matrix $S \in \mathcal{R}^{r \times r_{3}}$ such that $S^{\mathrm{T}} M S>0, S^{\mathrm{T}} C S \geq 0$, and $S^{\mathrm{T}} K S \geq 0$.

Proof. Since def $M=\operatorname{def}(M+C)>0$, there exists a matrix $U \in \mathcal{R}^{r \times r}$ such that $U^{\mathrm{T}} M U, U^{\mathrm{T}} C U$ and $U^{\mathrm{T}} K U$ are as given by (8). Define $S \in \mathcal{R}^{r \times r_{3}}$ by

$$
S=U\left[\begin{array}{c}
-K_{1}^{-1} K_{12} \\
I
\end{array}\right] \text {. }
$$


Then $S^{\mathrm{T}} M S=M_{2}$ is positive definite by ii) of Theorem 3 . Finally, $S^{\mathrm{T}} C S$ and $S^{\mathrm{T}} K S$ are nonnegative definite since $C$ and $K$ are nonnegative definite.

Remark. The matrix $S$ in Corollaries 2 and 3 gives the transformation that reduces the $r$-degree-of-freedom system (2) to a regular second-order system having fewer $\left(r_{3}\right)$ degrees of freedom. It is worth pointing out that if the conditions of Corollary 2 are satisfied, then the dimension of the state-space of the reduced system $\left(2 r_{3}\right)$ is less than the dimension of the subspace of consistent initial conditions $\left(2 r_{3}+r_{2}\right)$. In this case, the reduced system does not give solutions to all possible consistent initial conditions of the full system (2). However, it is often the case in applications that only the response of the reduced system is of interest. This response is completely determined by the initial conditions in the reduced state-space. This point will be illustrated in the examples. Finally, it should be noted that if $C=0$, then Corollary 3 reduces to the well-known Guyan reduction.

5. Examples. In this section, we present two examples to illustrate Theorem 3 and Corollary 2.

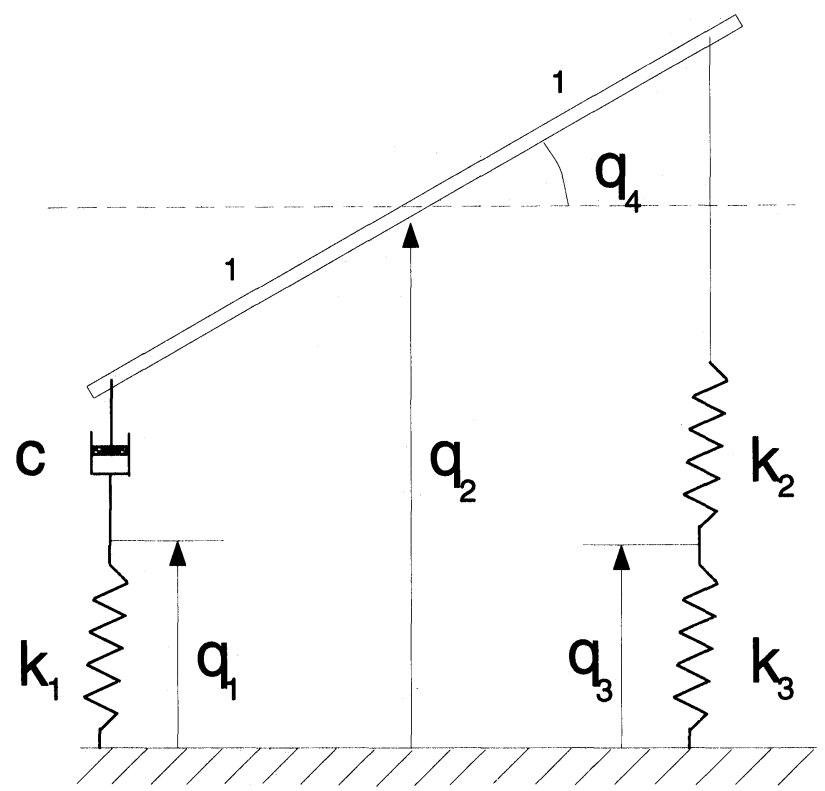

FIG. 1 .

Example 1. To illustrate Theorem 3, consider Figure 1, which shows a uniform rod of length 2 units having mass $m$ and moment of inertia $J$ about its center of mass. The motion of the rod takes place under the action of linear springs with positive spring constants $k_{1}, k_{2}$, and $k_{3}$ and a linear viscous damper with positive damping coefficient $c$ as shown. Assuming small motions, the unforced motion of this system is governed by (2) with

$$
M=\left[\begin{array}{cccc}
0 & 0 & 0 & 0 \\
0 & m & 0 & 0 \\
0 & 0 & 0 & 0 \\
0 & 0 & 0 & J
\end{array}\right], C=\left[\begin{array}{cccc}
c & -c & 0 & c \\
-c & c & 0 & -c \\
0 & 0 & 0 & 0 \\
c & -c & 0 & c
\end{array}\right]
$$




$$
K=\left[\begin{array}{cccc}
k_{1} & 0 & 0 & 0 \\
0 & k_{2} & -k_{2} & k_{2} \\
0 & -k_{2} & k_{2}+k_{3} & -k_{2} \\
0 & k_{2} & -k_{2} & k_{2}
\end{array}\right]
$$

and $q=\left[\begin{array}{llll}q_{1} & q_{2} & q_{3} & q_{4}\end{array}\right]^{\mathrm{T}}$. For this system $M+C+K>0, r_{1}=r_{2}=1$, and $r_{3}=2$. Letting $U$ be given by

$$
U=\left[\begin{array}{llll}
0 & 1 & 0 & 0 \\
0 & 0 & 1 & 0 \\
1 & 0 & 0 & 0 \\
0 & 0 & 0 & 1
\end{array}\right]
$$

so that $U^{\mathrm{T}} U=I$, it follows that

$$
\begin{aligned}
U^{\mathrm{T}} M U= & {\left[\begin{array}{cccc}
0 & 0 & 0 & 0 \\
0 & 0 & 0 & 0 \\
0 & 0 & m & 0 \\
0 & 0 & 0 & J
\end{array}\right], U^{\mathrm{T}} C U=\left[\begin{array}{cccc}
0 & 0 & 0 & 0 \\
0 & c & -c & c \\
0 & -c & c & -c \\
0 & c & -c & c
\end{array}\right], } \\
& U^{\mathrm{T}} K U=\left[\begin{array}{cccc}
k_{2}+k_{3} & 0 & -k_{2} & -k_{2} \\
0 & k_{1} & 0 & 0 \\
-k_{2} & 0 & k_{2} & k_{2} \\
-k_{2} & 0 & k_{2} & k_{2}
\end{array}\right] .
\end{aligned}
$$

This decomposition illustrates i) in Theorem 3.

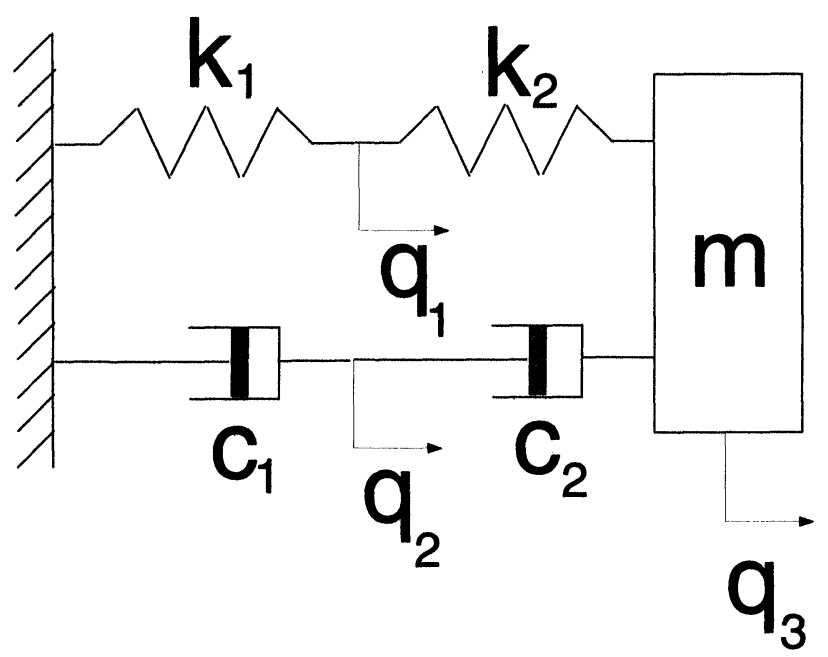

FIG. 2.

Example 2. Consider the lumped-parameter system shown in Figure 2 consisting of a mass $m$ with displacement $q_{3}$, linear springs with positive spring constants $k_{1}$ and $k_{2}$, and linear viscous dampers with positive damping coefficients $c_{1}$ and $c_{2}$. The massless joint between the dampers $c_{1}$ and $c_{2}$ has a displacement $q_{1}$, while the massless joint between the springs $k_{1}$ and $k_{2}$ has a displacement $q_{2}$. The equations of motion 
for this system can be written in the form (2) with

$$
\begin{gathered}
M=\left[\begin{array}{ccc}
0 & 0 & 0 \\
0 & 0 & 0 \\
0 & 0 & m
\end{array}\right], C=\left[\begin{array}{ccc}
0 & 0 & 0 \\
0 & c_{1}+c_{2} & -c_{2} \\
0 & -c_{2} & c_{2}
\end{array}\right], \\
K=\left[\begin{array}{ccc}
k_{1}+k_{2} & 0 & -k_{2} \\
0 & 0 & 0 \\
-k_{2} & 0 & k_{2}
\end{array}\right], q=\left[\begin{array}{l}
q_{1} \\
q_{2} \\
q_{3}
\end{array}\right] .
\end{gathered}
$$

It can easily be verified that $\mathcal{N}(M)=\operatorname{span}\left\{\left[\begin{array}{lll}1 & 0 & 0\end{array}\right]^{\mathrm{T}},\left[\begin{array}{lll}0 & 1 & 0\end{array}\right]^{\mathrm{T}}\right\}, \mathcal{N}(M+C)=$ $\operatorname{span}\left\{\left[\begin{array}{lll}1 & 0 & 0\end{array}\right]^{\mathrm{T}}\right\}$, and $\mathcal{N}(M+K)=\operatorname{span}\left\{\left[\begin{array}{lll}0 & 1 & 0\end{array}\right]^{\mathrm{T}}\right\}$. Thus the hypotheses of Corollary 2 are satisfied. The matrix $S$ in Corollary 2 is given by $S=\left[\begin{array}{lll}\frac{k_{2}}{k_{1}+k_{2}} & \frac{c_{2}}{c_{1}+c_{2}} & 1\end{array}\right]^{\mathrm{T}}$. The transformation $q=S \tilde{q}$ reduces (2) to

$$
m \ddot{\tilde{q}}+\frac{c_{1} c_{2}}{c_{1}+c_{2}} \dot{\tilde{q}}+\frac{k_{1} k_{2}}{k_{1}+k_{2}} \tilde{q}=0
$$

whose coefficients are consistent with the well-known formulas for series combinations of springs and dashpots. For this example, the subspace of consistent initial conditions has dimension 3. This follows from Corollary 1 by noting that $\operatorname{rank} M=1$ and $\operatorname{rank}(M+C)=2$. Thus only three independent quantities need to be specified at the initial instant, specifically, either $q_{2}, q_{3}$, and $\dot{q}_{3}$ or $q_{2}, \dot{q}_{2}$, and $q_{3}$. However, $q_{3}(t)=\tilde{q}(t)$ satisfies the reduced order equation (10) and is completely determined by the initial values of $q_{3}$ and $\dot{q}_{3}$. Consequently, $q_{3}(t)$ is independent of the initial value of $q_{2}$. In physical applications, the displacement of the mass is of primary interest. In such cases, the reduction procedure automatically eliminates the unwanted variable $q_{2}$. This illustrates the extension of Guyan reduction to systems with damping.

Acknowledgment. We wish to thank William Anderson and an anonymous reviewer for several helpful comments.

\section{REFERENCES}

[1] S. L. CAMPBell AND N. J. Rose, Singular perturbation of autonomous linear systems, SIAM J. Math. Anal., 10 (1979), pp. 542-551.

[2] S. L. CAMPBELL, Optimal control of autonomous linear processes with singular matrices in the quadratic cost functional, SIAM J. Control Optim., 14 (1976), pp. 1092-1106.

[3] P. BERNHARD, On singular implicit linear dynamical systems, SIAM J. Control Optim., 20 (1982), pp. 612-633.

[4] S. L. CAmpbell ANd N. J. Rose, A second order singular linear system arising in electric power systems analysis, Internat. J. Systems Sci., 13 (1982), pp. 101-108.

[5] R. J. GUYAN, Reduction of stiffness and mass matrices, AIAA J., 3 (1965), p. 380.

[6] J. Argyris ANd H. Mlejnek, Dynamics of Structures, North-Holland, Amsterdam, 1991.

[7] S. K. AGRAWAL, Inertia matrix singularity of series-chain spatial manipulators with point masses, J. Dynamic Systems, Measurement, Control, 115 (1993), pp. 723-725.

[8] D. CоBB, On the solutions of linear differential equations with singular coefficients, J. Differential Equations, 46 (1982), pp. 310-323.

[9] S. L. CAMPBell AND C. D. MEYeR JR., Generalized Inverses of Linear Transformations, Pitman, Boston, MA, 1979.

[10] S. L. CAmpbelL, Singular Systems of Differential Equations, Pitman, Boston, MA, 1980. 